\title{
Reliability Approach in View of Damping in MEMS Resonator
}

\author{
${ }^{\square}$ Poorvi K. Joshi
}

Department of Electronics Engineering, Shri Ramdeobaba College of Engineering and Management, Nagpur (MS), India.

joshipk@rknec.edu

Received: 08th May 2020, Accepted: 15th July 2020, Published: 31st August 2020

\begin{abstract}
MEMS resonators are important component in most of the RF applications. For reliable operation Quality factor should be high and important in transceivers for synchronizing and better sensitivity. This paper presents overview of work done in the area of energy dissipation due to damping in MEMS resonator and to understand techniques which can helps in minimizing its effect. It also suggests an approach to study the different damping mechanisms and analysis of reliability in view of aging and performance through accelerated testing.
\end{abstract}

Keywords

Reliability, MEMS Resonator, Damping, Quality Factor

\section{Introduction}

Damping mechanism is very important in MEMS based devices. Understanding and quantification of damping effects is main issue in determining the sensitivity of the device and depends on the higher quality factor (Q). Understanding of energy loss mechanism is important aspect in designing of the MEMS resonators.

Quality factor is important in transceivers for synchronizing, better sensitivity and for reliable operation Q-factor should be high. MEMS resonators operates for fixed resonant frequency and is most important characteristics, any shift in it, affects the performance of the device and suitability for specific application. Quality factor is a ratio of maximum energy (total energy) stored to energy dissipated (lost) in one cycle as given in equation 1.

$Q=2 \pi \frac{\text { Energy Stored }}{\text { Energy Dissipated }}_{\text {Per Cycle }}$

So by minimizing the energy losses, Q-factor of resonator can be improved. Various mechanisms associated with energy loss, contributing in overall quality factor are classify into three broad categories; viz. fluid-structure interaction, boundary damping and material damping as given in Table 1.

Fluid-structural damping occurs in resonator chamber having gas or liquid, energy dissipation occurs due to transfer of structural energy in thermal energy of molecule of fluids during every cycle of vibration. Fluidstructural interaction is associated with viscous damping, squeeze film damping (SFD), acoustic radiation and internal flow. Fluid-structure damping depends on fluidic properties, material properties, size, mode, temperature, frequency, pressure and geometry [1]. With advancement in micromachining technology, on chip micro resonators can produce high Q-factor if operated in vacuum to reduce losses due to fluidic damping. In air damping, air molecules are source to transfer energy from vibrating structure. SFD occurs due to air or gas trapped between MEMS structure and substrate. SFD is very much affected by boundary conditions, structure geometry, fluid film thickness, oscillation frequency and packaging pressure. Movement of mechanical components produces sound waves which creates acoustic radiation source of damping mechanism.

Boundary damping mechanism is related to elastic wave radiation (stress wave) causes support losses or anchor losses from resonator to frame supporting the structure, microsliding occurs at resonator to frame to package and viscoelasticity arises in gel and adhesive used in bonding frame with package. Clamping losses can be referred to elastic wave radiation or microsliding [1]. Energy loss transmission to supporting structure of the vibrating structure is clamping loss which is irretrievable.

Material damping is classified as Thermoelastic damping (TED) which occurs due to irreversible heat conduction causes elastic defomation, Akhiezer damping due to phonon-phonon interaction, phonon-electron interaction and internal friction causes due to various defects [1]. Applied strain field results in relaxation of temperature due to which energy lost referred as TED. Energy loss through thermo-elastic dissipation (TED) occurs when heat irreversibly flows across the temperature gradient caused by elastic deformation in resonating structures.

Table 1: Categorization of Damping in MEMS Resonator

\begin{tabular}{|l|l|l|}
\hline Fluid structure interaction & Material Damping & Boundary Damping \\
\hline Viscous & Thermo Elastic (TED) & Elastic wave radiation (Stress wave) \\
\hline Squeeze Film & Phonon-phonon (Akhieser) & Anchor Loss \\
\hline Acoustic & Phonon-electron & Microsliding (Clamping) \\
\hline Air & Internal Friction & Viscolelasticity \\
\hline Internal Flow & & \\
\hline
\end{tabular}


The coefficient of thermal expansion plays a vital role in this process as regions of a resonator in compression due to increase in temperature and tension when cools down [2]. Various damping mechanisms limits the value of quality factor. Mechanical energy dissipation is important issue in MEMS resonator [3]. Energy dissipation measurement models are available for few damping mechanisms mentioned earlier. Finite-element and finite difference methods or closed-form expression techniques are used for controlling the various damping mechanisms and provide design guidelines [1]

Damping is the most important parameter to be considered while designing miniaturized structures of MEMS devices. Energy loss in MEMS based devices occurs due to various mechanisms is the key issue. Energy losses from various damping mechanisms in parallel is calculated in terms of total Q-factor as given in equation 2, where $i$ is different loss mechanisms.

$\frac{1}{Q_{\text {Total }}}=\sum_{i} \frac{1}{Q_{i}}$

Various parameters control the magnitude of damping are modes of vibration, type of structure, type of material, defects, microstructure, residual stresses, supports, clamps and packaging etc. There can be other parameters like external source operation in different energy domain controlling damping in oscillation [1]. Damping is assessed using harmonic excitation, free decay, wave propagation and thermomechanical noise by monitoring structural dynamics [1].

Dissipation can be the reliability parameter because all these dissipations are indirectly converted into heat and the device performance. Commercialization of MEMS resonator depends on the reliability and sustainability under various operating conditions and environment. Reliability and reproducibility of device is most critical issue in design of MEMS resonator under various dynamic inputs. Sensitivity, robustness, stability and resilience are key performance indicator of MEMS resonator under variable loads. Reliability is very important aspect in order to enhance the application of MEMS devices for different applications. Various technical issues due to small size of MEMS resonator are fatigue and temperature fluctuation which can be caused due to damping which directly affects the aging and shift in frequency. Damping mechanism is important parameter to be considered while doing reliability testing.

This paper presents overview of work done in area of energy dissipation due to damping in MEMS resonator and to understand various techniques which can helps in minimizing its effect.

The aim of this review is to suggest an approach to understand importance of various damping mechanism and its effect on aging, performance by reliability testing. It also suggests an approach to study the different damping mechanisms and analysis of reliability in view of aging and performance through accelerated testing of MEMS Resonator.

\section{Damping}

Identification of various regions of energy dissipation and suggesting modification in MEMS resonator design due to fluid-structure interaction, boundary damping and material damping mechanism are very well elaborated by researchers and is briefed in following sections.

\section{Fluid-Structure Interaction}

D. D. Gerrard et al [2] analyzed the TED region in order to reduce the energy loss by changing geometry of MEMS resonator structure and its effect on quality factor limit. They suggested that identification regions of major rate of heat flow facilitate MEMS designer to improve design through geometric modification and enhance Qfactor. S.A. Chandorkar et al [4] formulated the entropy based TED for three dimensional heat transfers and estimation of energy loss in various resonator structures. With this technique estimation of Q-factor is carried out in bulk, flexural and torsional mode resonator structure. They proposed that entropy based formulation is better in estimating Q-factor and required less time. Chengzhong Ma et al. [5] presented 2-D thermal conduction model to obtained TED by application of uniform radial pre-tension stress on circular resonator. They reported that pretension stress increases the resonant frequency and reduced TED. Thermal conduction along thickness of circular plate and in radial direction considered while modelling. Also they discussed the effect of thickness to radius ratio, dimensions and modes on TED. They concluded that pre-tension makes resonator stiffer, so store more mechanical energy and energy dissipation due to TED is changed.

\section{Material Damping}

J. Rodriguez et al [3] examined various mechanisms which limit the quality factor in MEMS resonator. They carried out experimentation to estimate the anchor damping and quality factor in MEMS resonator. They developed a method to mitigate all the energy dissipation sources.and investigated the dependency of anchor damping on anchor architecture and on material used for mounting. Weinberg et al [6] reviewed energy loss mechanisms in MEMS resonator and its impact on gyros and radio frequency resonator. They discussed the thermal energy loss due to phonon interaction, elaborated about TED and Akhieser effect in MEMS resonators. They also deliberated on support effects and losses, fluid losses and electric damping and impact of high Q-factor on performance of MEMS gyro and RF resonator. S. A. Chandorkar et al [7] analyzed the quality factor limits for 
bulk mode MEMS resonators and material selection choice for achieving high quality factor. Estimated limits for Q-factor in bulk mode resonator for three modes of operation; lame, wine glass and extensional mode. They observed that energy loss is due to TED limits the Q-factor in lame mode as structure developed compression and tension region during operation. In wine glass and extensional mode Q-factor limit was set by Akheiser effect. They show that the Q-factor and frequency (Q- $f$ ) product for silicon is slightly higher than quartz resonator and silicon carbide can achieved twenty times higher Q-f product. J. Rodriguez et al [8] quantify the Q-factor of silicon lame mode MEMS resonator in variation of temperature and compare Q-factors for various designs and anchors. They clearly detected the Akhiezer damping in MEMS resonator by measurement of Q-factor and its temperature dependence. D. D. Gerrard et al. [9] presented topology optimization approach for minimizing TED in MEMS resonator. Finite element analysis was used to model 2D plane stress TED and COMSOL was used for validation. They concluded that topology optimization can help in developing new design to increase Q-factor in TED limited devices. Yoneoka et al [10] presented characterization results of MEMS resonator which was fabricated and packed in a poly $\mathrm{SiC}$ with thin film encapsulation. They evaluated the energy loss mechanisms on performance and impact of poly $\mathrm{SiC}$ coating on frequency with respect to temperature. They observed that pressure inside the package can be controlled and frequency drifts of resonator are less than frequency noise level from experimentation. Stresses induced due to change in temperature and various factors affecting the TC are resonator material and dimensions. Estimated TED from experiment results was less than the theoretical model, the difference occurs due to the contribution of other energy loss mechanism in the system. Xin Zhou et al. [11] presented strategy to mitigate TED for flexural mode micromechanical resonator. They suspended lumped masses on frame structure to reduced TED in micromechanical flexural mode resonator by separating resonant frequency from thermal relaxation rate. They proposed that this approach will improve stability, sensitivity and resolution of resonator and perfect for designing high performance MEMS gyroscope resonator. A. L. Alter et al. [12] investigated the nonlinear dissipation for MEMS flexural mode resonator operated in nonlinear regime. Ringdown Experimentation was performed in different pressure and high vacuum in order to isolate different damping sources. They compared TED and gas damping for different combinations to study the origin of nonlinear dissipation in MEMS resonator. They found that nonlinear dissipation is more substantial in pressure limited resonator than the high vacuum, and TED and gas damping contribution is significant.

\section{Boundary Damping}

J. Rodriguez et al [13] measured the quality factor of MEMS resonator under different temperature range and also compute the energy loss mechanisms due to anchor damping. At temperature $-155^{\circ} \mathrm{C}$ energy loss due to TED, air, akhieser damping are minimum and only anchor damping influence on Q-factor. Experimentation performed with variation in conduction medium between silicon die mounted to ceramic package and relationship of Q-factor Vs temperature was plotted. With repetitive experiments they found that mounting between die and package effectively trap the energy and validate accuracy and repeatability of results. A. Darvishian et al. [14] investigated anchor loss mechanism in hemispherical shell resonator (HSR) by classifying study based on shell properties, properties of stem, effect due to substrate characteristics and external motion effects. They analyzed that all propagating waves passing from stem to substrate are captured by perfectly match layer (PML) acts as absorbing layer. They found that anchor loss in HSR is mainly depends on shell, stem, substrate and motion effects. They concluded that for controlling anchor damping in HSR, precise manufacturing process was important parameter than the design parameters. H. J. Kim et al. [15] studied the anchor loss and TED affecting the flicker frequency $(1 / f)$ noise on aluminum nitride (AIN) piezoelectric MEMS resonator. They designed and manufactured 104 AlN contour mode resonator (CMR) half of them are from different chip for varied range of Q-factor and by varying resonator parameter analyzed the noise and damping relationship. They concluded that making of ultra-low noise MEMS resonator for various applications, understanding the effect of damping on $1 / f$ noise is important. J. Segovia-Fernandez [16] modelled the anchor damping and TED mechanism in AIN contour mode resonator, also estimated Q-factor at very high and ultra-high frequency. Semi analytical 1D approach was used for qualitative estimation of $\mathrm{Q}_{\mathrm{ted}}$ and for anchor loss fixed constraint technique was used for predicting $\mathrm{Q}_{\mathrm{anc}}$. Author found that TED depends on thermal property of metal layer. J. Rodriguez et al. [17] developed new method to measure Qfactor at low temperature to accurately locate the energy dissipation from anchor damping in MEMS tuning fork resonator (single and double anchored). They also studied the impact of anchor design and die mounting structure on anchor damping. They measured anchor damping in single and double anchored tuning fork resonator which depends on the structure connecting die with system. They found that the energy escape is retain in die can be returned to the resonator thus increases Q-factor for the resonator. G. D. Vukasin et al. [18] found that reducing the thickness of substrate of MEMS tuning fork resonator reduces the anchor damping. They performed the experimentation for the resonator anchored to thin and thick bottom substrate to observed relationship between anchor damping and die thickness. They measured $\mathrm{Q}(\mathrm{T})$ and concluded that the anchor damping is less in thin die than the thicker.

\section{All Category Damping}

B. Kim et al [19] analyzed the various energy loss mechanisms, their temperature dependence with respect to air damping, TED, anchor and surface loss and explore effect on Q-factor in MEMS resonator. Temperature 
coefficient of Q-factor (TCQ) was used to understand the magnitude of temperature dependence of Q-factor. As Q-factor has strong dependence on temperature, it can be used as temperature measuring device to measure temperature of resonator directly and instantly for close loop control. M. Jandak et al [20] investigated the energy loss mechanisms in clamp-clamp (CC) and clamp-free (CF) beam resonator and estimated Q-factor experimentally and compared it with analytical models. Experimentation is carried out by varying the geometry of resonator under low pressure. They found that variation in length over wide range easily distinguish between the TED and anchor damping effect, also the change in temperature affects the overall Q-factor. M. Gologanu et al [21] presented analytical and finite element method (FEM) model for estimating and measuring energy losses due to TED, acoustic radiation and viscous fluid damping. They concluded that 3D FEM is better tool for analyzing various energy loss mechanisms in complex structures. G. D. Vukasin et al [22] measured Q-factor owing to anchor, gas damping and TED of a ring resonator and analyzed the Q-factor under pressure and temperature. For centre-anchored and dual-side anchored resonator they compared anchor damping. They found that damping mechanism identification and estimation is best possible with measurement of Q(T) over a range of temperature in MEMS resonator. They concluded that anchor damping is strong for Centre anchored than double anchored resonator.

\section{Motivation and Approach}

There are various factors affecting the response due to variation in resonant frequency like; technological aspects, principle of operation, geometry, material properties, ambient conditions, environmental factors (temperature, pressure and damping) etc. Thermal Stability is drift in resonance frequency with change in temperature. In order to develop reliable product with its expected in-use lifetime, every steps in manufacturing is to be recorded systematically and categorized the root cause of failures anticipating the failure mode. This will help in achieving better MEMS resonator reliability and disciplined approach during designing, manufacturing and quality checks minimizes the risk of failures.

This paper aims to suggest an approach to study the different damping mechanisms and tests for analysis of reliability of MEMS resonator. Approach consist of selection of high frequency MEMS resonator and designing of testing circuit to carry out different accelerated testing for analyzing performance analysis and characterization. Reliability testing aims to prolong the useful life and predict start of aging by analyzing various failure and damping mechanism. Simulation will be used for analyzing the functioning based on the test predictions and suggest suitable modification in process of design and fabrication of the MEMS resonator.

\section{Discussion}

Damping is important issue in MEMS resonator to understand its effect and also to quantify its severity on vibrating structure. Increase in Q-factor leads to increases in sensitivity of device. Various studies and analysis on energy dissipation mechanism due to damping in MEMS resonator and its mitigation techniques are brief in this work. In view of reliability analysis of MEMS resonator study of energy dissipation is key issue to understand importance of various damping mechanism and its effect on aging, performance by reliability testing. Various parameters caused due to damping which directly affects the aging and shift in frequency needs to be understood and quantify. It will be useful for improving the design of MEMS resonator for better reliability and sustainability.

\section{Conclusion}

In previous decade requirement of high quality factor for RF applications motivates researchers to minimize the energy dissipation in MEMS resonators by analyzing and controlling various damping mechanisms. Advance modeling techniques can be used to identify energy loss mechanism. Material properties like coefficient of thermal expansion is relationship between temperature and volume of material influences TED. In Akheiser effect Q-f product is only based on material property and not dependent on structural geometry. Die mounting plays significant role in high Q-factor resonator and in minimization of energy loss due to anchor damping. Energy loss mechanisms due to defect damping, bulk dislocation, surface roughness and composite structure can be explored. Validation and limitation of various damping analysis models can be further explored in more detail.

\section{References}

[1] S. Joshi, S. Hung and S. Vengallatore, "Design Strategies for controlling damping in micromechanical and nanomechanical resonators”, EPJ Techniques and Instruments, 1, 5 (2014), pp: 1-14, doi: 10.1186/epjti5

[2] D. D. Gerrard et al., "Manipulation of heat flux paths in thermo-elastically damped resonators for Q optimization," 2017 IEEE 30th International Conference on Micro Electro Mechanical Systems (MEMS), Las Vegas, NV, 2017, pp. 1130-1133, doi: 10.1109/MEMSYS.2017.7863613

[3] J. Rodriguez, S. A. Chandorkar, G, M. Glaze, D. D. Gerrard, Y. Chen, D. B. Heinz, I. B. Flader, and T. W. Kenny, "Direct Detection of Anchor Damping in MEMS Tuning Fork Resonators", Microelectromechanical Systems, 2018, VOL. 27, doi:10.1109/jms.2019.2859958 
[4] S. A. Chandorkar, R. N. Candler, A. Duwel, R. Melamud, M. Agarwal, K. E. Goodson, T. W. Kenny, "Multimode thermoelastic dissipation", Journal of Applied Physics, 2009, Vol. 105, issue 4, doi: $10.1063 / 1.3072682$

[5] Chengzhong Ma, Siyu Chen and Fenglin Guo, "Thermoelastic damping in micromechanical circular plate resonators with radial pre-tension", Journal of Thermal Stresses, Vol 43, Issue 2, pp: 175-190, DOI: 10.1080/01495739.2019.1652714

[6] M. Weinberg, R. Candler, S. Chandorkar, J. Varsanik, T. Kenny and A. Duwel1, "Energy loss in MEMS resonators and the impact on inertial and RF devices," TRANSDUCERS 2009 - 2009 International Solid-State Sensors, Actuators and Microsystems Conference, Denver, CO, 2009, pp. 688-695, doi: 10.1109/SENSOR.2009.5285418

[7] S. A. Chandorkar, M. Agarwal, R. Melamud, R. N. Candler, K. E. Goodson and T. W. Kenny, "Limits of quality factor in bulk-mode micromechanical resonators," 2008 IEEE 21st International Conference on Micro Electro Mechanical Systems, Wuhan, 2008, pp. 74-77, doi: 10.1109/MEMSYS.2008.4443596

[8] J. Rodriguez et al. "Direct Detection of Akhiezer Damping in a Silicon MEMS Resonator". Science Report 9, 2244 (2019), doi: 10.1038/s41598-019-38847-6

[9] D. D. Gerrard et al., "Topology optimization for reduction of thermo-elastic dissipation in MEMS resonators," 2017 19th International Conference on Solid-State Sensors, Actuators and Microsystems (TRANSDUCERS), Kaohsiung, 2017, pp. 794-797, doi: 10.1109/TRANSDUCERS.2017.7994168

[10] S. Yoneoka et al., "Characterization of Encapsulated Micromechanical Resonators Sealed and Coated With Polycrystalline SiC," in Journal of Microelectromechanical Systems, vol. 19, no. 2, pp. 357-366, April 2010, doi: 10.1109/JMEMS.2010.2040460

[11] Xin Zhou et al., "Mitigating Thermoelastic Dissipation of Flexural Micromechanical Resonators by Decoupling Resonant Frequency from Thermal Relaxation Rate", Physical Review Applied, 2017, Vol 8, issue 6, pp: 064033(8 pages), doi: 10.1103/PhysRevApplied.8.064033.

[12] A. L. Alter et al., "Experimentally Observed Nonlinear Dissipation Linked to Contributions from Gas Damping and TED in Mems Flexural Mode Resonators," 2019 20th International Conference on Solid-State Sensors, Actuators and Microsystems \& Eurosensors XXXIII (Transducers \& Eurosensors XXXIII), Berlin, Germany, pp. 2095-2098, doi: 10.1109/TRANSDUCERS.2019.8808777

[13] J. Rodriguez et al., "Direct measurements of anchor damping in MEMS resonators," 2017 IEEE SENSORS, Glasgow, 2017, pp. 1-3, doi: 10.1109/ICSENS.2017.8233870

[14] A. Darvishian, B. Shiari, J. Y. Cho, T. Nagourney and K. Najafi, "Anchor Loss in Hemispherical Shell Resonators," in Journal of Microelectromechanical Systems, vol. 26, no. 1, pp. 51-66, Feb. 2017, doi: 10.1109/JMEMS.2016.2636080

[15] H. J. Kim, J. Segovia-Fernandez and G. Piazza, "Damping directly impacts flicker frequency noise of piezoelectric aluminum nitride resonators," 2016 IEEE 29th International Conference on Micro Electro Mechanical Systems (MEMS), Shanghai, 2016, pp. 667-670, doi: 10.1109/MEMSYS.2016.7421714

[16] J. Segovia-Fernandez, "Damping in aluminum nitride contour mode MEMS resonators," 2017, IEEE 60th International Midwest Symposium on Circuits and Systems (MWSCAS), Boston, MA, 2017, pp. 49-52, doi: 10.1109/MWSCAS.2017.8052857

[17] J. Rodriguez et al., "Unanticipated results in the first direct measurements of anchor damping in MEMS resonators," 2018 IEEE Micro Electro Mechanical Systems (MEMS), Belfast, 2018, pp. 543-546., doi: 10.1109/MEMSYS.2018.8346610

[18] G. D. Vukasin et al., "Effect of Substrate Thickness on Anchor Damping in MEMS Devices," 2019, 20th International Conference on Solid-State Sensors, Actuators and Microsystems \& Eurosensors XXXIII (TRANSDUCERS \& EUROSENSORS XXXIII), Berlin, Germany, pp. 1843-1845, doi: 10.1109/TRANSDUCERS.2019.8808424

[19] B. Kim et al., "Temperature Dependence of Quality Factor in MEMS Resonators," in Journal of Microelectromechanical Systems, vol. 17, no. 3, pp. 755-766, June 2008, doi: 10.1109/JMEMS.2008.924253

[20] M. Jandak, T. Neuzil, M. Schneider, U. Schmid, "Investigation on different damping mechanisms on the Q factor of MEMS resonators", 2016, Procedia Engineering, vol. 168, pp. 929-932, doi: 10.1016/j.proeng.2016.11.308

[21] M. Gologanu, C. G. Bostan, V. Avramescu and O. Buiu, "Damping effects in MEMS resonators," CAS 2012 (International Semiconductor Conference), Sinaia, 2012, pp. 67-76, Doi: 10.1109/SMICND.2012.6400695.

[22] G. D. Vukasin et al. "Direct Measurements Of Anchor Damping In Pressure-Limited Ring Resonators," Conference Proceeding, 2018, pp: 370-371, doi:10.31438/trf.hh2018.105 